\title{
Distribution of Cardiac Myosin Isozymes in Human Conduction System Immunohistochemical Study Using Monoclonal Antibodies
}

\author{
Makoto Kuro-o, Hidetsugu Tsuchimochi, Seigo Ueda, Fumimaro Takaku, and Yoshio Yazaki \\ Third Department of Internal Medicine, Faculty of Medicine, University of Tokyo, Hongo, Bunkyo-ku, Tokyo 113, Japan
}

\begin{abstract}
To determine the presence and distribution of cardiac myosin isozymes in the human conduction system, we performed an immunohistochemical study using monoclonal antibodies CMA19 and HMC14, which are specific for myosin heavy chains of human atrial type ( $\alpha$-type) and ventricular type ( $\beta$-type), respectively. Serial frozen sections of human hearts were obtained from autopsy samples and examined by indirect immunofluorescence. $\alpha$-type was found in all myofibers of sinus node and atrio-ventricular node, and in $55.2 \pm \mathbf{1 0 . 2} \%$ (mean $\pm \mathrm{SD}, n=5$ ) of the myofibers of ventricular conduction tissue, which consists of the bundle of His, bundle branches, and the Purkinje network. In contrast, $\beta$-type was found in all myofibers of the atrio-ventricular node and ventricular conduction tissue, whereas almost all myofibers of the sinus node were unlabeled by HMC14. Although the number of ventricular myofibers labeled by CMA19 was small, the labeled myofibers were more numerous in the subepicardial region than in the subendocardial region. These findings show that the gene coding for $\alpha$-type is expressed predominantly in specialized myocardium compared with the adjacent ordinary working myocardium.
\end{abstract}

\section{Introduction}

Recent studies have demonstrated myosin heterogeneity in cardiac muscle. Two types of cardiac myosin heavy chains $\left(\mathrm{HC}^{1}\right.$ and $H C \beta$ ) encoded by distinctive genes (1) are regarded as comprising three myosin isozymes designated as $\mathrm{V} 1, \mathrm{~V} 2$, and $\mathrm{V} 3$, which correspond to homodimers of $\mathrm{HC} \alpha$, heterodimers of $\mathrm{HC} \alpha$, and $H C \beta$, and homodimers of $H C \beta$, respectively (2). V1 has higher ATPase activity than V3 (3-5), and V3 has increased efficiency of force production compared with V1 $(6,7)$. Isoforms of myosin heavy chain show characteristic patterns in their distribution according to species $(3,4)$, fiber type (8-10), age $(11,12)$, hormonal state $(4,12,13)$, and pressure overload (1418). Especially, isozymic changes induced by pressure overload are thought to play some role in the pathophysiology of cardiac hypertrophy (16-18). However, myosin isozymes of human car-

A part of this work was presented at the American Heart Association's 57th Scientific Session (1984. Circulation. 70:II-78. [Abstr.]).

Address reprint requests to Dr. Kuro-o, University of Tokyo.

Received for publication 17 May 1985 and in revised form 8 October 1985.

1. Abbreviations used in this paper: AV, atrio-ventricular, ELISA, enzymelinked immunosorbent assay; $\mathrm{HC} \alpha$, heavy chain $\alpha ; \mathrm{HC} \beta$, heavy chain $\beta$; LC, light chain.

\section{J. Clin. Invest.}

(c) The American Society for Clinical Investigation, Inc.

0021-9738/86/02/0340/08 $\$ 1.00$

Volume 77, February 1986, 340-347 diac muscle are difficult to separate by the electrophoretic method, and even the existence of isozymes was not confirmed until quite recently. Monoclonal antibodies have been widely used in various fields because of the advantages that $(a)$ homogeneous antibodies can be obtained infinitely, and $(b)$ they are suitable for discriminating slight differences in protein conformation. We have paid attention to these advantages and have succeeded in developing monoclonal antibodies specific for human cardiac myosin heavy chains of either atrial type $(\mathrm{HC} \alpha)$ or ventricular type (HC $\beta)$. By means of an immunohistochemical method using these monoclonal antibodies, we have demonstrated the existence and distribution of myosin isozymes in human atrium (17).

Although knowledge about myosin isozymes in ordinary working myocardium has accumulated as described above, there are few reports concerning those in specialized myocardium which composes the conduction system. By immunohistochemical methods, Sartore et al. (10) showed myosin polymorphism in Purkinje fibers of chicken atrium, and variable reactivity of Purkinje fibers with anti-bovine atrial myosin antibody in the bovine ventricle (8), but the presence and distribution of myosin isozymes in the whole conduction system has not been elucidated, especially in humans. By using monoclonal antibodies, we have now demonstrated the detailed distribution of myosin isozymes in normal human myocardium including the conduction system.

\section{Methods}

Myosins were isolated from human ventricles and bovine atria by a dilution technique as previously described (3). The light chains (I and II) were isolated from the myosin molecule by guanidine denaturation (19). Monoclonal antibodies specific for $\mathrm{HC} \alpha$ or $\mathrm{HC} \beta$ were obtained from cloned hybridomas as previously reported (17). In brief, hybridomas producing anti-myosin antibodies were obtained by fusion of mouse myeloma cells $(\mathrm{P} 3 \times 63 \mathrm{Ag} 8 \mathrm{U} 1)$ with isolated spleen cells of $\mathrm{BALB} / \mathrm{c}$ (male, 6w) mice immunized with bovine atrial or human ventricular myosin $(0.1-0.2 \mathrm{ml}$ of a $1-\mathrm{mg} / \mathrm{ml}$ myosin solution, injected intraperitoneally at 2-wk intervals) as $\mathrm{HC} \alpha$ or $\mathrm{HC} \beta$ antigen, respectively, essentially according to the protocol of Köhler and Milstein (20). Anti-myosin activity in the medium from hybridoma colonies was screened by enzymelinked immunosorbent assay (ELISA) by the method reported by Guesdon et al. (21). The ELISA test has been described in detail elsewhere (17). Briefly, myosin and light chains $(100 \mu \mathrm{g} / \mathrm{ml}$ in phosphate-buffered saline [PBS]) were bound to each well of a 96-well microtiter plate and blocked with $1 \%$ bovine serum albumin solution. Medium from the hybridoma colonies was reacted with bound antigens for $1 \mathrm{~h}$ at $37^{\circ} \mathrm{C}$, then, after three washings with PBS, biotinylated goat anti-mouse immunoglobulin (Vector Laboratories Inc., Burlingame, CA) was added to each well. After incubation for $1 \mathrm{~h}$ at room temperature, the wells were washed three times with PBS containing $0.5 \%$ Tween-20, then avidin D-peroxidase (Vector Laboratories) was added with $\mathrm{H}_{2} \mathrm{O}_{2}, 4$ amino-antipyrin, and phenol as substrate. Optical density at $550 \mathrm{~nm}$ was measured. ELISA tests with light chains as antigens were performed in order to determine whether these antibodies react with heavy chains or light chains. 

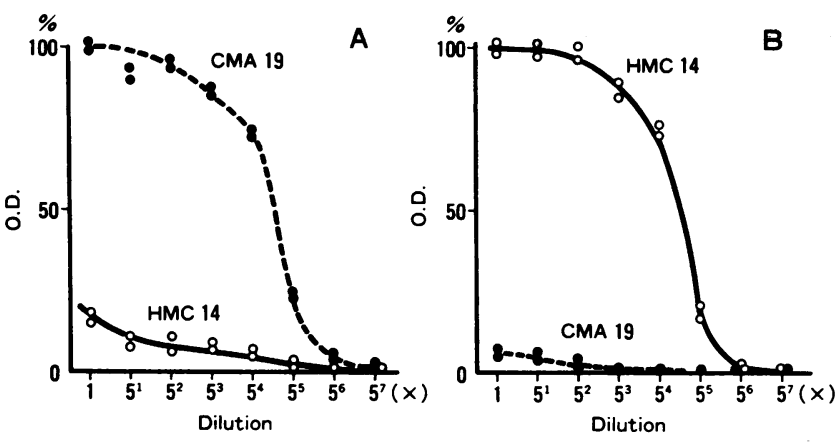

Figure 1. Reactions of the monoclonal antibodies with human atrial $(A)$ and ventricular $(B)$ myosin. Results are expressed as the percentage of maximum optical density at $550 \mathrm{~nm}$. CMA19 (•) and HMC14 (O) reacted specifically with atrial and ventricular myosin, respectively. They show no reaction with light chains. The negligible reaction between CMA19 and ventricular myosin, and the reduced amount of cross-reactivity between $\mathrm{HMC} 14$ and atrial myosin are explained by the presence of a very small quantity of atrial-type myosin in the ventricle, and a significant amount of ventricular-type myosin in the atrium, respectively.

Specimens of human heart were obtained at autopsy from five subjects without heart disease. Several blocks of cardiac muscle containing conduction system and cross sections of ventricle were excised within $3 \mathrm{~h}$ after death. Excision of conduction tissue was carried out according to the methods described by Davies et al. (22). The blocks for the sinus node enclosed the crista terminalis and the superior caval vein. The plane of section was set perpendicular to the sulcus terminalis. The blocks for the atrio-ventricular (AV) node and ventricular conduction tissue extended from the orifice of the coronary sinus to the membranous septum and contained both the interatrial and interventricular septum. The plain of section was perpendicular to the atrio-ventricular junction made through the membranous septum. The cross section of the whole ventricle was divided into three transmural blocks; i.e., left and right ventricular free wall and interventricular septum. The blocks were embedded in Tissue Tek II O.C.T. compound (Miles Laboratories Inc., Naperville, IL) and immediately frozen in liquid nitrogen. Serial cryostat sections were prepared for immunofluorescence. Parts of them were fixed with methanol for 5-10 min and stained by Masson-Goldner staining in order to identify the conduction tissues.

For indirect immunofluorescence, sections were first incubated with the anti-myosin monoclonal antibodies for $1 \mathrm{~h}$ at $37^{\circ} \mathrm{C}$. Then the sections were rinsed in $10 \mathrm{mM}$ PBS, and treated with biotinylated goat antimouse IgG (Tago Laboratories Inc., Burlingame, CA) for $1 \mathrm{~h}$ at $37^{\circ} \mathrm{C}$. They were again rinsed in PBS, and finally stained with fluorescein isothiocyanate-labeled avidin (EY Laboratories Inc., San Mateo, CA) for $1 \mathrm{~h}$ at $37^{\circ} \mathrm{C}$. All antibodies were diluted appropriately with PBS containing $0.04 \%$ sodium azide. The sections were mounted in glycerol and examined with a microscope with epifluorescence optics (Nikon Inc. Instrument Div., Garden City, NY). In order to determine the amount of $\mathrm{HC} \alpha$ or $\mathrm{HC} \beta$ more quantitatively, the percentage of labeled myofibers was calculated for each case.
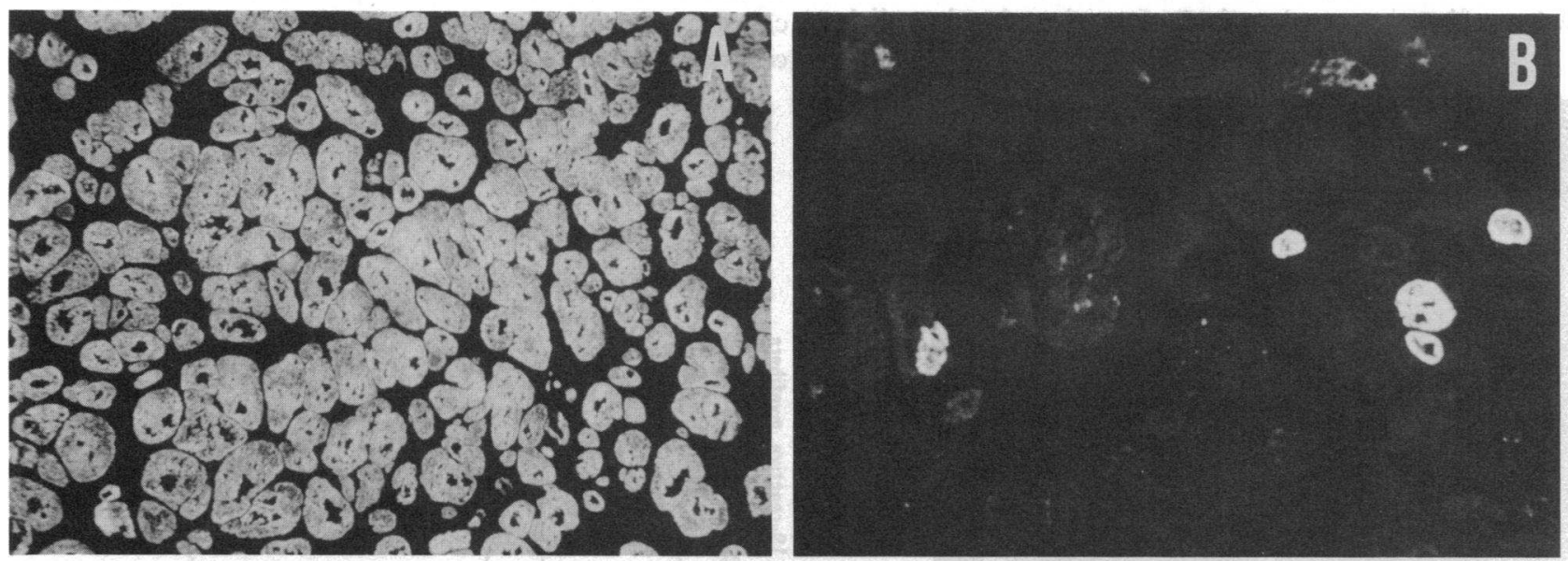

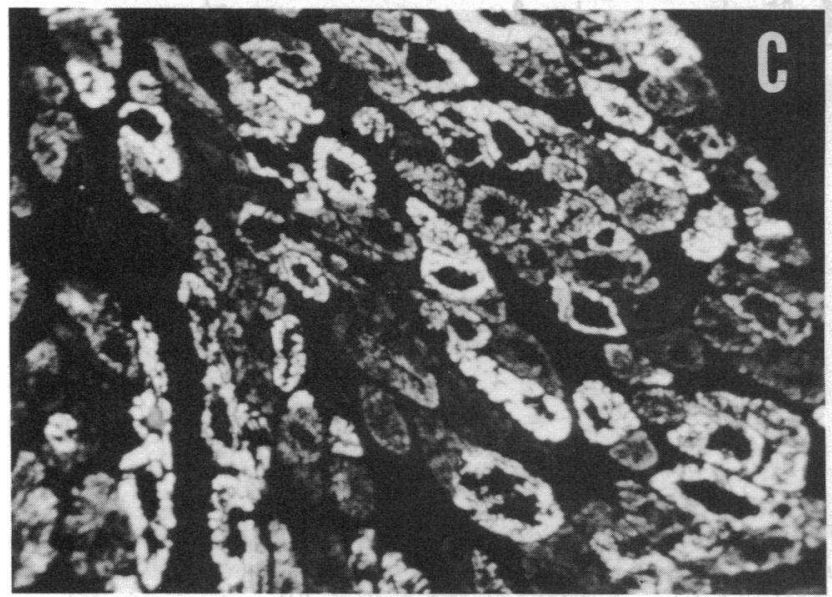

Figure 2. (A) Cryostat section of normal human ventricle stained with HMC14. All myofibers reacted strongly. (B) As in $A$, except that the section was stained with CMA19. A small number of fibers are labeled. $(C)$ Cryostat section of normal human atrium stained with

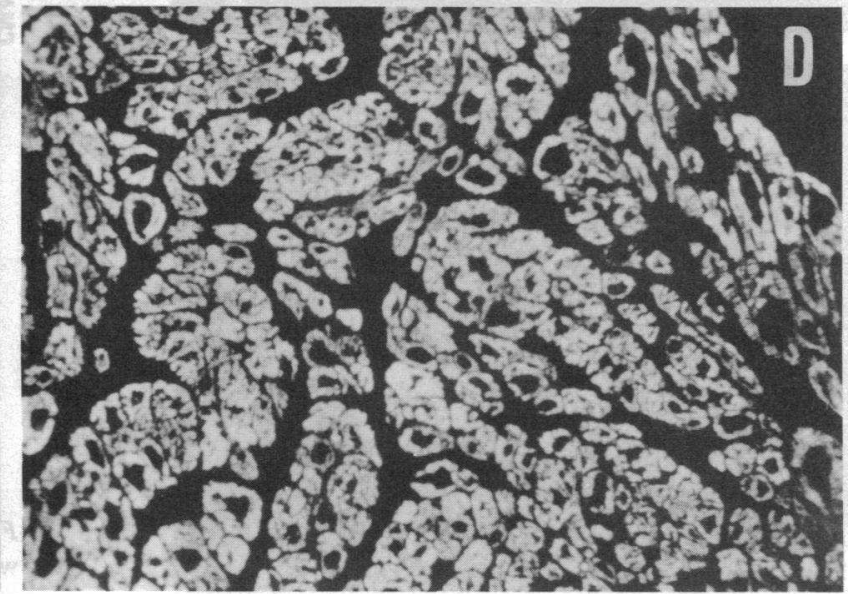

HMC14. Some myofibers were unreactive. Note that the intensity of staining of labeled myofibers is highly variable. $(D)$ As in $C$, except that the myofibers were stained with CMA19. Almost all myofibers are strongly labeled. 


\section{Results}

Two clones (CMA19 and HMC14) of hybrid cells secreting antimyosin antibodies were selected for discriminating the antigenic difference between $\mathrm{HC} \alpha$ and $\mathrm{HC} \beta$. The characteristics of these antibodies were described in our previous report (17). Briefly, in the ELISA tests, CMA19 reacted with the atrial myosin specifically, and HMC14 selectively reacted with the ventricular myosin. Neither CMA19 nor HMC14 reacted with light chains of these cardiac myosins (Fig. 1).

In the immunofluorescence study, these two antimyosin antibodies were found to stain atrial and ventricular myocardium in completely different patterns (Fig. 2). When HMC14 was applied to sections of ventricular myocardium, all muscle fibers reacted strongly and homogeneously (Fig. $2 A$ ). However, when it was applied to sections of atrial appendage, some myofibers were unreactive (Fig. $2 C$ ). In contrast, almost all myofibers of the atrial appendage were strongly labeled by CMA19 (Fig. 2 $D$ ), whereas all except a few of the ventricular myofibers were unreactive (Fig. 2 B). No difference in the reactivity with CMA19 and $\mathrm{HMCl} 4$ was observed between the right and left ventricle, while the number of atrial myofibers labeled by $\mathrm{HMCl} 4$ tended to be more numerous in the left atrium than in the right atrium. The proportion of the ventricular myofibers labeled by CMA 19 was relatively small, but showed significant regional variation (Fig. 3). Hardly any of the muscle fibers just under the endocardium were labeled by CMA19 (Fig. 3 End). The number of labeled myofibers increased gradually from the subendocardial
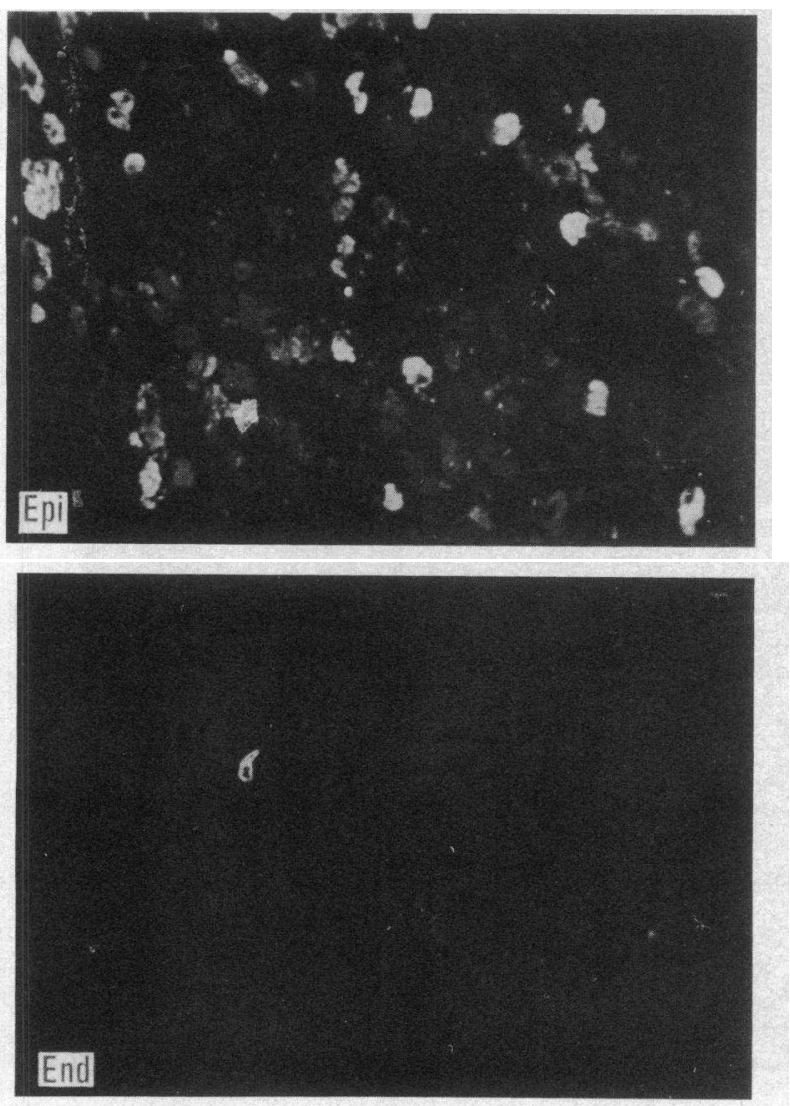

Figure 3. Ventricular myocardium stained with CMA19. (Epi) A section from the subepicardial region. A greater number of myofibers is labeled than in panel End. (End) A section from the subendocardial region. Almost all myofibers are unlabeled. to the subepicardial region, where up to $15 \%$ of the myofibers were stained by CMA19 (Fig. 3 Epi). Interestingly, a considerable number of papillary myofibers were also labeled, even though they were located just next to the subendocardial ventricular myocardium. In Fig. 4, we demonstrate a typical case showing the distribution of labeled muscle fibers revealed by CMA19 as described above.

In the conduction tissue, the response to these antibodies was quite different from that of the ordinary working myocardium. When the antimyosin antibodies were applied to the sections of the sinus node, all myofibers were strongly stained by CMA19 (Fig. $5 \mathrm{~A}$ ), whereas only a minor proportion (mean $\pm \mathrm{SD}$; $4.7 \pm 1.8 \%, n=5$ ) was reactive with $\mathrm{HMCl} 4$ (Fig. 5 B). A striking difference in reactivity was observed between myofibers of the sinus node and those of the surrounding ordinary atrial myocardium. The latter was predominantly labeled by $\mathrm{HMC14}$, and especially in the crista terminalis next to the sinus node, almost all muscle cells were reactive (Fig. $5 B$ ).

In the AV node, all muscle fibers were stained by HMC14 homogeneously (Fig. $6 \mathrm{~A}$ ), whereas the reactivity with CMA19 did not show a homogeneous pattern. In the central portion of the AV node and the transitional zone between the atrial myocardium and the AV node, all muscle fibers were reactive with CMA19 (Fig. 6 B). However, the number of labeled myofibers decreased in the transitional zone between the AV node and the bundle of His. As soon as the myofibers of the AV node began to penetrate into the annulus fibrosus, the staining pattern changed suddenly and the proportion of the CMA19-reactive cells decreased to about one-half (Fig. $6 C$ ). In the atrial septum

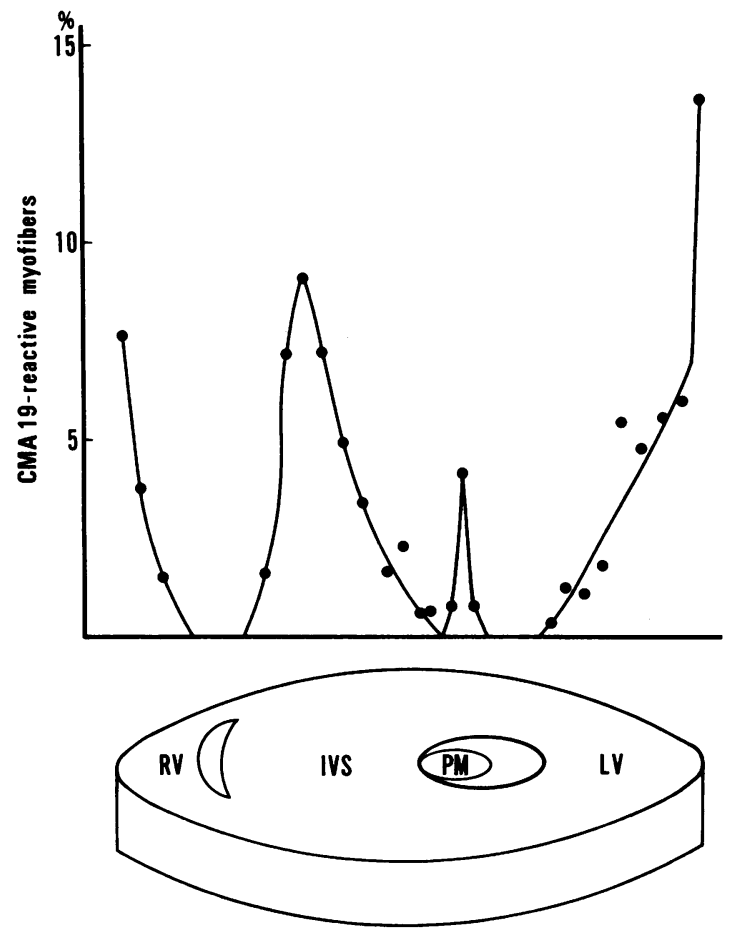

Figure 4. Scheme of the distribution of ventricular myofibers reactive with CMA19. The number of labeled myofibers is greater in the subepicardial region and the central portion of the interventricular septum than in the subendocardial region. Note that papillary myofibers also show regional variation in the number of labeled fibers. RV, right ventricle; IVS, interventricular septum; LV, left ventricle; PM, papillary muscle. 

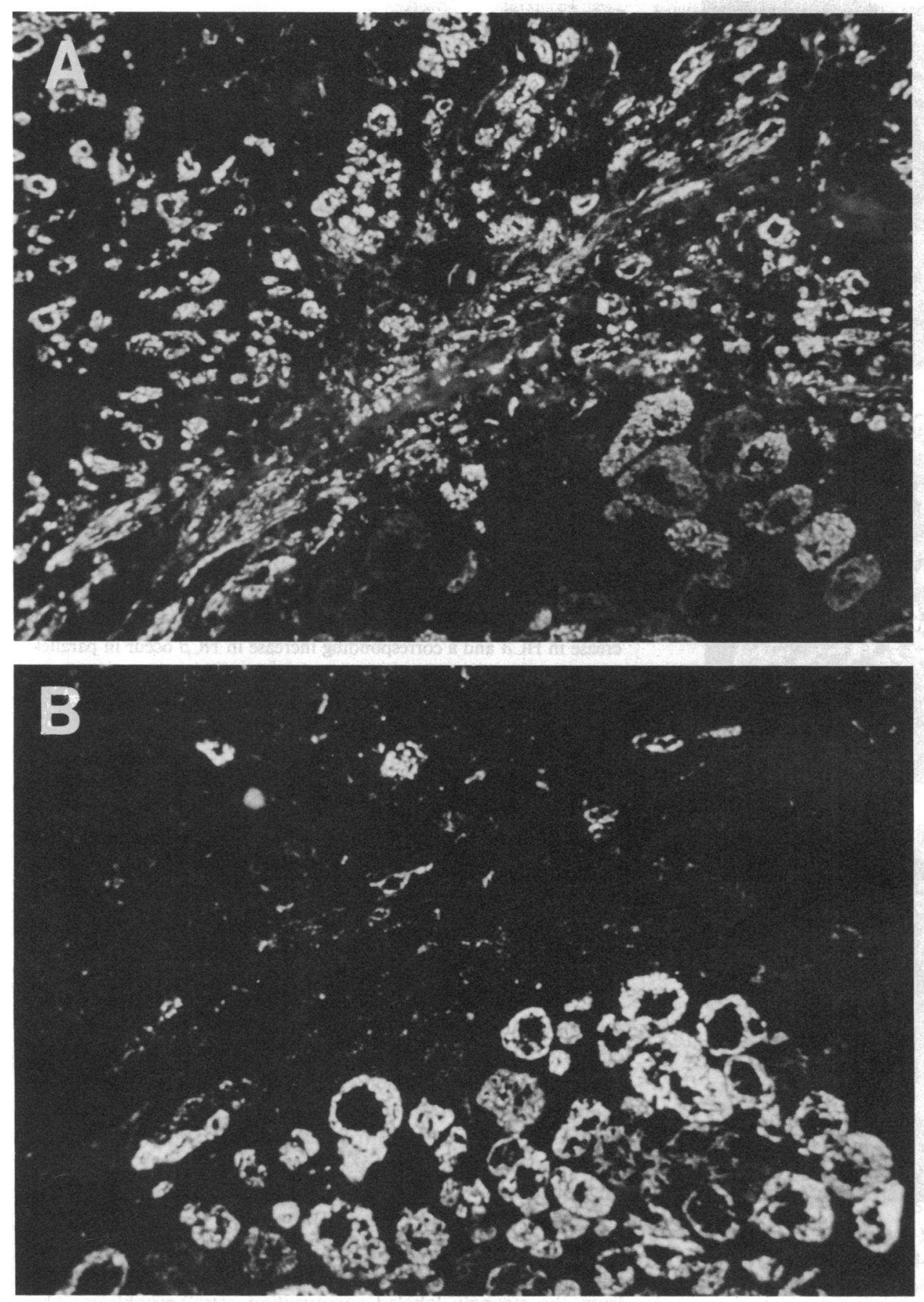

Figure 5. Cryostat sections of human sinus node. (A) Stained with

CMA19. Sections were comprised of sinus node (upper field, smaller cells) and crista terminalis (lower field, larger cells). All myofibers of the sinus node are strongly labeled, while a few myofibers of the crista terminalis are unlabeled. (B) Stained with HMC14. Note the clear contrast in reactivity between sinus node and crista terminalis. Almost all myofibers of the crista terminalis are labeled, and form a clear contrast to those of the sinus node. next to the AV node, almost all myofibers were reactive not only with CMA19 but also with HMC14. The proportion of atrial myofibers labeled by HMC14 was greater in the crista terminalis and the lower portion of the atrial septum than in the appendage.

In the bundle of His, all muscle fibers were stained by HMC14 homogeneously (Fig. $7 \mathrm{~B}$ ), and a considerable proportion of myofibers (mean $\pm \mathrm{SD} ; 55.2 \pm 10.2 \%, n=5$ ) were also reactive with CMA19 (Fig. $7 A$ ). The intensity of staining by CMA19 varied from completely negative to strongly positive, and gave the appearance of a mosaic pattern (Fig. $7 \mathrm{~A}$ ).

Bundle branches and Purkinje fibers showed the same reactivity with both antibodies as the bundle of His (Fig. 8, $A$ and $B)$. No difference was observed between right and left bundle branches. A striking difference in reactivity with CMA19 between
Purkinje fibers and ordinary ventricular myocardium is demonstrated in Fig. $8 \mathrm{~A}$. When sections of ventricular conduction tissues were stained with CMA19, Purkinje fibers were seen as a bundle labeled in a mosaic pattern at the endocardial surface that formed a clear contrast to the unlabeled ordinary ventricular myofibers in the subendocardial region.

The distribution of isoforms of myosin heavy chain in specialized and ordinary working myocardium described above is summarized in Table I.

\section{Discussion}

As previously reported from our laboratory, almost all muscle fibers of the human atrium contain $\mathrm{HC} \alpha$ and $20-60 \%$ of them 

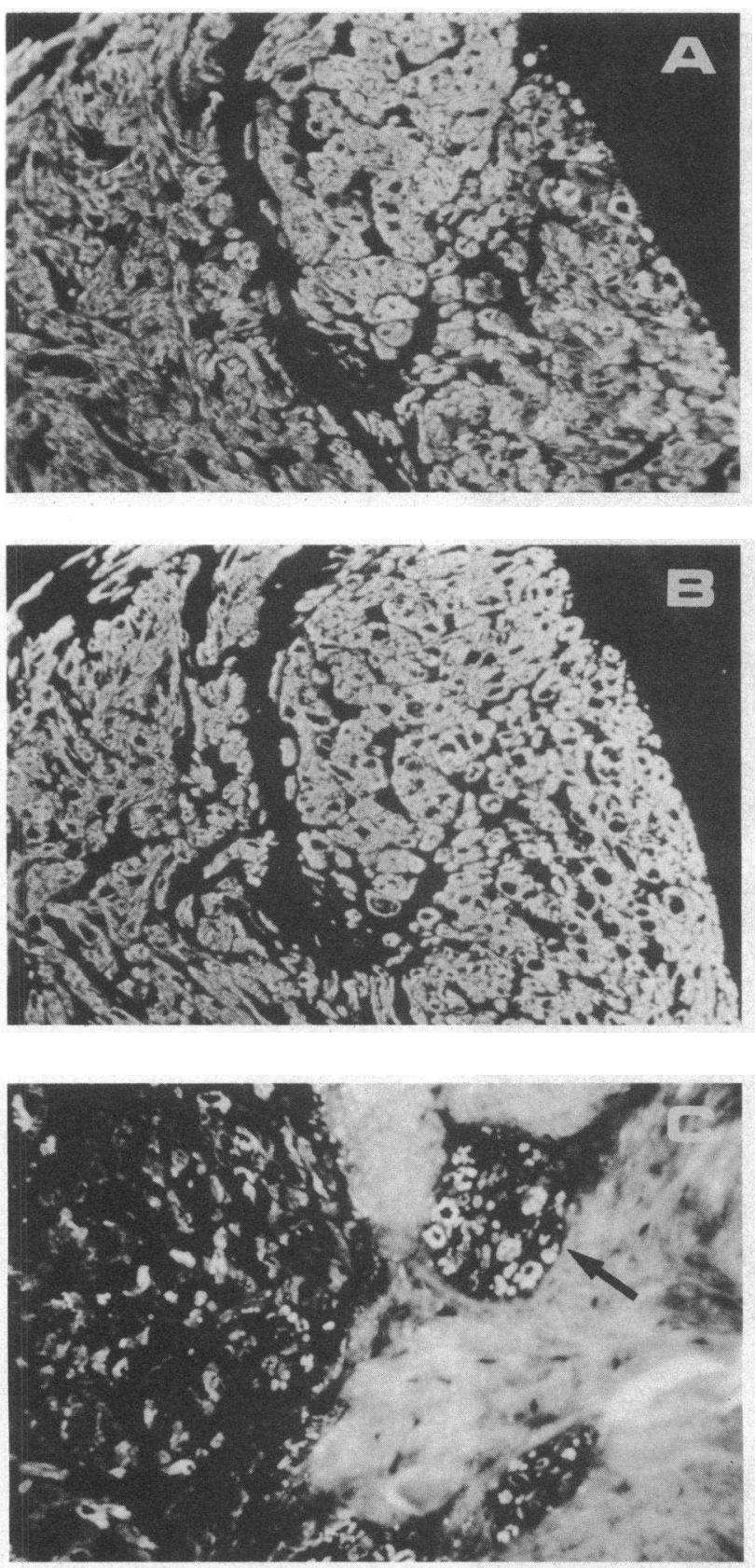

Figure 6. Cryostat sections of human AV node. $(A)$ The central portion of the AV node stained with HMC14. All myofibers are strongly reactive. (B) As in $A$, except that the section was stained with CMA19. All myofibers are also labeled. $(C)$ The transitional zone between AV node and the bundle of His stained with CMA19. Note that the proportion of labeled fibers has decreased to about one-half in myofibers of the AV node penetrating into the annulus fibrosus (arrow), and show a similar staining pattern to that in the bundle of His.

also contain $\mathrm{HC} \beta$. In contrast, all muscle fibers of the human ventricle contain $\mathrm{HC} \beta$, while only a few of them contain $\mathrm{HC} \alpha$ (17). The present study demonstrates (a) significant regional variation in the number of ventricular myofibers containing $\mathrm{HC} \alpha,(b)$ the existence of myosin isozymes in the human conduction system, and $(c)$ a striking difference in their distribution between specialized and ordinary working myocardium.

As almost all myofibers of the crista terminalis and the interatrial septum were labeled by $\mathrm{HMC14}$, they are abundant in
Table I. Distribution of Isoforms of Myosin Heavy Chain in Human Cardiac Muscle

\begin{tabular}{lll}
\hline Fiber type & $\begin{array}{l}\text { CMA19-reactive } \\
\text { myofibers }\end{array}$ & $\begin{array}{l}\text { HMC14-reactive } \\
\text { myofibers }\end{array}$ \\
\hline & $\%$ & $\%$ \\
Sinus node & 100 & $4.7 \pm 1.8^{*}$ \\
$\begin{array}{l}\text { Atrium } \\
\text { AV node } \\
\text { Bundle of His } \\
\text { Bundle branches }\end{array}$ & 100 & $20-100 \ddagger$ \\
$\begin{array}{l}\text { Purkinje fibers } \\
\text { Ventricle }\end{array}$ & 100 & 100 \\
\hline
\end{tabular}

* Mean $\pm \mathrm{SD}, n=5$.

$\ddagger$ The proportion of labeled myofibers showed significant regional variations. In the atrium, almost all myofibers of the crista terminalis and the lower portion of the interatrial septum were labeled by HMC14, whereas only $20-60 \%$ of the myofibers in the appendage were labeled. In the ventricle, almost all myofibers in the subendocardial region were unlabeled by CMA19, whereas up to $15 \%$ of the myofibers in the subepicardial region were labeled. Note that a decrease in $\mathrm{HC} \alpha$ and a corresponding increase in $\mathrm{HC} \beta$ occur in parallel with the order of the electrical excitation, that is, the decrease in intrinsic frequency of spontaneous discharge.

HC $\beta$ compared with the other portions of the atrium such as the appendage. These findings are consistent with those observed in the bovine heart by Gorza et al. (18). However, we did not observe the tendency for the HC $\beta$-containing cells to be more numerous in the right atrium than in the left atrium, as reported by them. On the contrary, we saw more $\mathrm{HC} \beta$ in the left than in the right atrium. Considering that the mean pressure in the normal atrium is higher in the left than in the right atrium, and that $\mathrm{HC} \beta$ appears to be suited for pressure work, our results are reasonable from the standpoint of pressure overload. As the specific antibody for $\mathrm{HC} \beta$, they used anti-human pectoralis myosin antiserum, which was absorbed by bovine left atrial myosin (18), while we used a monoclonal antibody obtained from mice immunized with human ventricular myosin. The reason for the inconsistency is not clear, as we stated previously (17), but it might be due to the different characteristics of the anti-myosin antibodies.

In contrast, almost all myofibers of the sinus node were not labeled by $\mathrm{HMC14}$, forming a striking difference in reactivity from the strongly labeled surrounding atrial myofibers. This shows that the sinus node contains hardly any of the ventricular type myosin heavy chain that exists in the crista terminalis adjoining them.

Almost all myofibers of the AV node were found to contain both $\mathrm{HC} \alpha$ and $\mathrm{HC} \beta$ within single cells. This allows two different interpretations in terms of myosin isozymes: the isomyosins exist as (1) V2 (heterodimers of $\mathrm{HC} \alpha$ and $\mathrm{HC} \beta$ ) or (2) a mixture of $\mathrm{V} 1$ and $\mathrm{V} 3$ (homodimers of $\mathrm{HC} \alpha$ or $\mathrm{HC} \beta$, respectively) within single cells. With our immunohistochemical method, it is impossible to determine which is true.

In ventricular conduction tissue, all muscle fibers contain $\mathrm{HC} \beta$ as in the adjoining ordinary ventricular myocardium in the subendocardial region, whereas the proportion of $\mathrm{HC} \alpha$-containing cells presents a striking contrast between them. Ventricular myocardium, especially in the subendocardial region, con- 

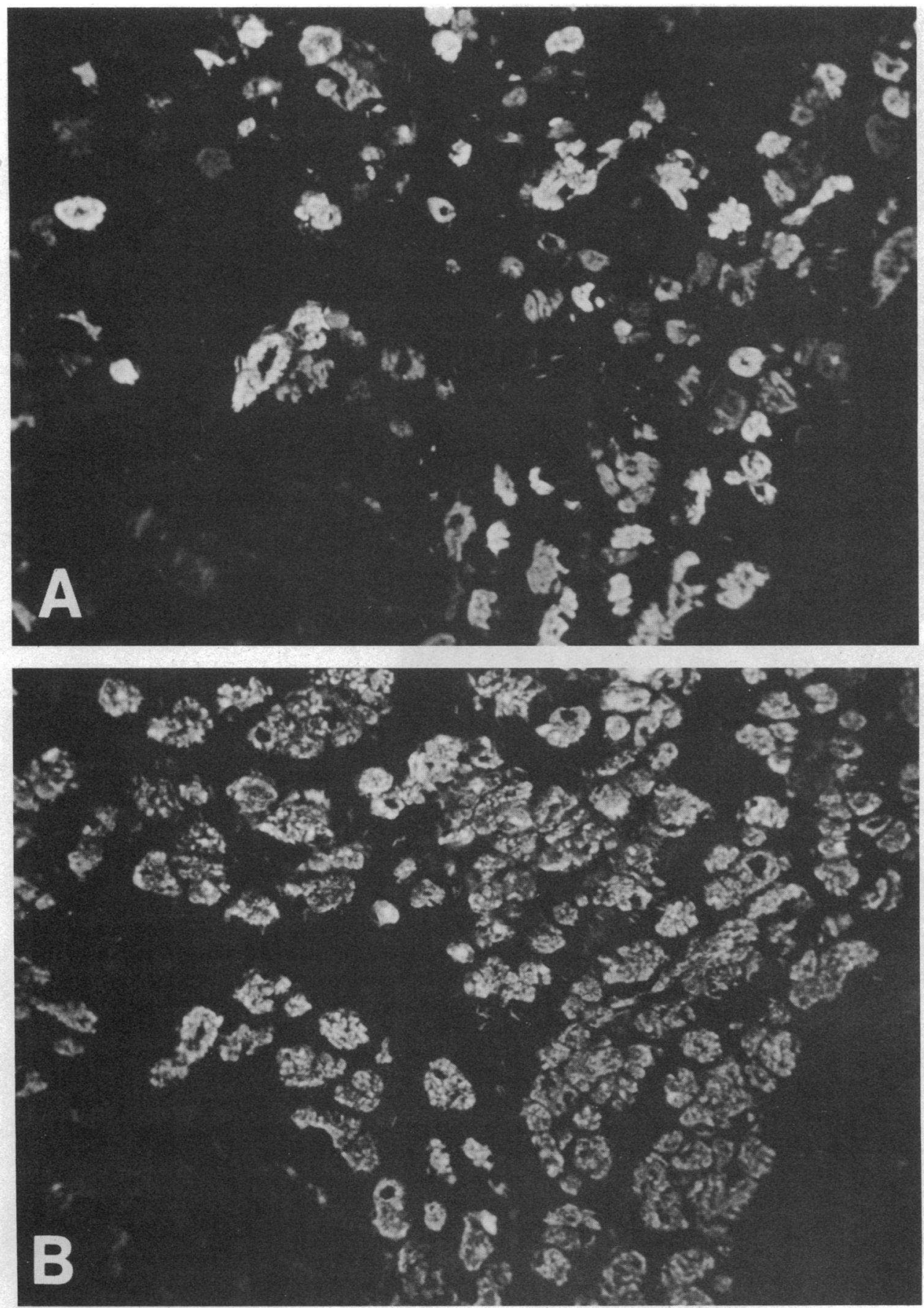

Figure 7. Serial cryostat sections of the bundle of His. (A) Stained with CMA19. About one-half of the myofibers are labeled. Note that the staining intensity is highly variable from completely negative to strongly positive staining. (B) Stained with HMC14. All myofibers are labeled homogeneously.

tains hardly any $\mathrm{HC} \alpha$, while up to one-half of the myocytes in the His-Purkinje system were found to contain $\mathrm{HC} \alpha$. Consequently, it is quite probable that the subendocardial myofibers labeled by CMA19 are those of ventricular conduction tissue. The relatively high $\mathrm{HC} \alpha$ content in the Purkinje network is consistent with previously reported findings for the bovine Purkinje fibers (8). Thornell et al. (23) and Tamura et al. (24) demonstrated that myosin ATPase activity is higher in the Purkinje fibers than in the ordinary ventricular myocardium in bovine heart. Considering that V1 shows higher ATPase activity than V3 (3-5), their results are in good agreement with the finding of a higher HC $\alpha$ content in Purkinje cells. Saito et al. (25) reported that myosin from bovine ventricular conduction tissue gives three bands of light chains ( $\mathrm{LC1}, \mathrm{LCl}^{\prime}$, and $\mathrm{LC} 2$ ) in sodium dodecyl sulfate-polyacrylamide gel electrophoresis, and that LC1 and LC2 show the same mobility as the light chains of ordinary ventricular myocardium. They recently reported that $\mathrm{LCl}^{\prime}$ shows the same mobility as atrial LCl (26). Their results concerning light chains are very interesting from the viewpoint that the ventricular conduction tissue contains considerable amounts of myosin heavy chains of atrial type ( $\mathrm{HC} \alpha)$.

The significant difference in the distribution of ventricular myofibers containing $\mathrm{HC} \alpha$ between the subendocardial and the subepicardial region is considered to correspond to the difference in the contractile conditions between them; myofibers in the subendocardial region are required to perform greater pressure work than those in the subepicardial region. Since V3 shows greater economy of force production than V1 $(6,7), \mathrm{HC} \beta$ appears 

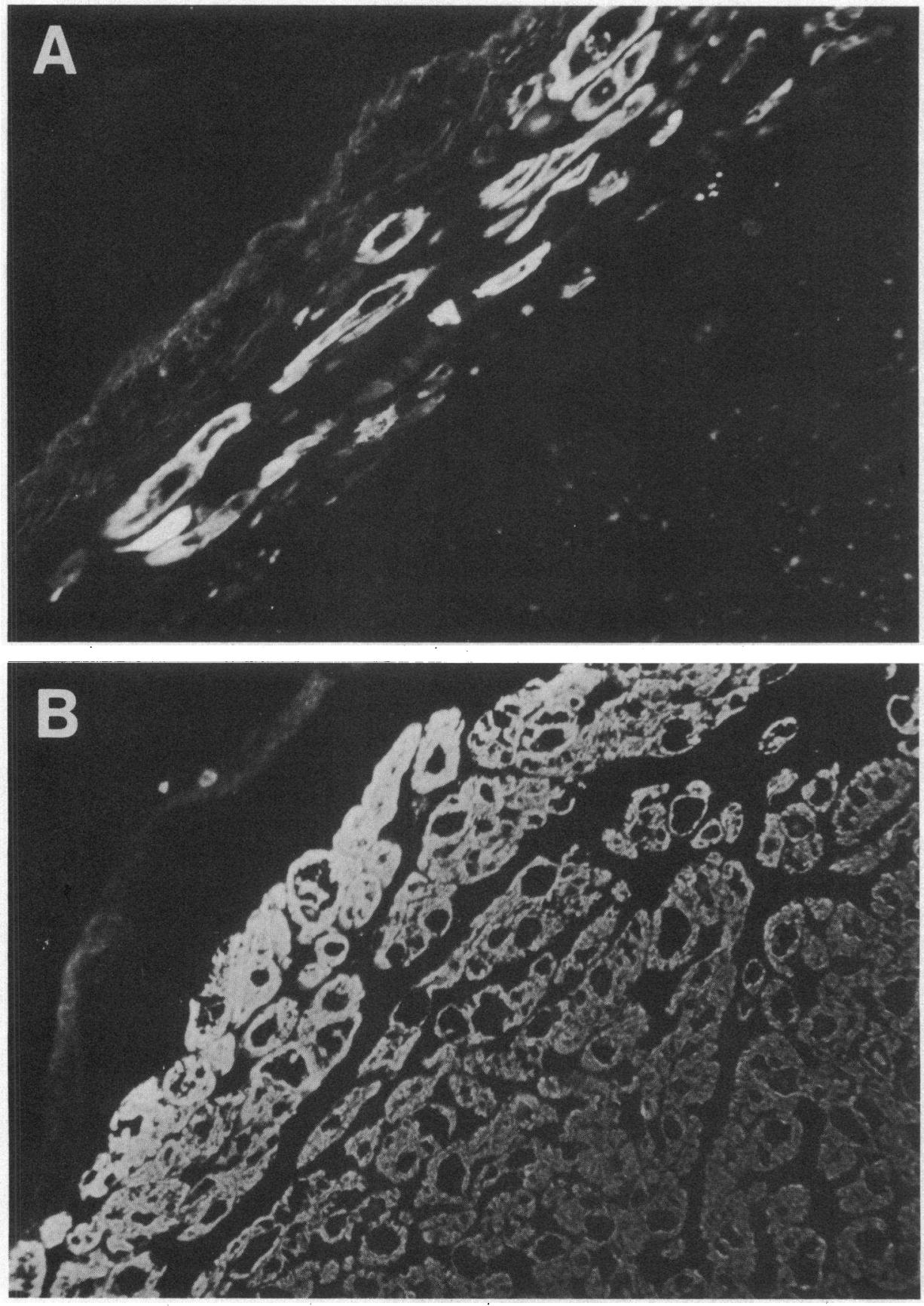

Figure 8. Cryostat sections of Purkinje fibers and ventricular myocardium. $(A)$ Stained with CMA19. Purkinje fibers occur as a labeled bundle at the very surface of the endocardium, and show the same reactivity as myofibers of the bundle of His (Fig. 7 A). Note the striking difference in reactivity between Purkinje fibers and ordinary working ventricular myocardium. (B) Stained with HMC14. All myofibers are labeled. to be a physiological isoform of myosin heavy chain for performing pressure work. In fact, we have demonstrated a decrease in the amount of $\mathrm{HC} \alpha$ with a corresponding increase in $\mathrm{HC} \beta$ in pressure-overloaded human atria (17), and Gorza et al. (18) reported a decrease in $\mathrm{HC} \alpha$ in pressure-overloaded human ventricle. Therefore, the regional variation shown here is regarded as a physiological adaptation to the wall stress.

For the same reason, the relatively high $\mathrm{HC} \alpha$ content of myofibers of ventricular conduction tissue suggests that the HisPurkinje system might be spared such pressure work as would cause isozymic transformation from $\mathrm{V} 1$ to $\mathrm{V} 3$, whereas it is located in the subendocardial region where myofibers are exposed to the greatest wall stress. Thornell and co-workers (23) reported that Purkinje fibers in the bovine heart contain a large number of intermediate filaments performing a cytoskeletal function. It is likely that the abundant cytoskeleton diminishes mechanical stress on the myofibrils in Purkinje fibers and allows the high $\mathrm{HC} \alpha$ content

In our present study, as all the conduction cells were labeled by CMA 19 or HMC14 or both, there is no evidence for the existence of another myosin isozyme different from $\mathrm{HC} \alpha$ and $\mathrm{HC} \beta$ in the human conduction system. On the other hand, Gonzalez-Sanchez and Bader (27) reported that a monoclonal antibody directed against myosin heavy chains of the anterior latissimus dorsi in the chicken cross-reacts with the Purkinje fibers in the adult and late-embryonic chicken heart in immunofluorescence tests. This suggests that there are some structural differences between myosin heavy chains of the chicken Purkinje fiber and ordinary myocardium. However, as they stated in their paper, their results do not necessarily mean the existence of an- 
other myosin isozyme specific for the Purkinje fibers. Whether there is an isozyme other than $\mathrm{HC} \alpha$ and $\mathrm{HC} \beta$ in the human conduction system remains to be clarified.

Another interesting finding is that the proportion of $\mathrm{HC} \alpha-$ containing cells decreases in parallel with the decrease in the intrinsic rate of spontaneous pacemaker discharge (Table I). Further investigations are needed to determine the physiological significance of this distinctive distribution of myosin isozymes in specialized myocardium.

\section{Acknowledgments}

We wish to thank Prof. T. Shirai, Department of Pathology, and Prof. K. Okumura, Department of Immunology, Juntendo University School of Medicine, Tokyo, Japan, for their kindness in providing human hearts and for valuable discussion, and Dr. S. Ohkawa, Department of Internal Medicine, Tokyo Metropolitan Geriatric Hospital, Tokyo, Japan, for helpful suggestions in making sections of the conduction system.

This study was supported in part by a grant-in-aid for scientific research from the Ministry of Education, Science and Culture, and by a grant from the Ministry of Health and Welfare, Japan.

\section{References}

1. Mahdavi, V., A. P. Chambers, and B. Nadal-Ginard. 1984. Cardiac $\alpha$-and $\beta$-myosin heavy chain genes are organized in tandem. Proc. Natl. Acad. Sci. USA. 81:2626-2630.

2. Hoh, J. F. Y., P. A. McGrath, and P. J. Hale. 1978. Electrophoretic analysis of multiple forms of rat cardiac myosin: effect of hypophysectomy and thyroxine replacement. J. Mol. Cell. Cardiol. 10:1053-1076.

3. Yazaki, Y., and M. S. Raben. 1974. Cardiac myosin adenosinetriphosphatase of rat and mouse: distinctive enzymatic properties compared with rabbit and dog cardiac myosin. Circ. Res. 35:15-23.

4. Yazaki, Y., and M. S. Raben. 1975. Effect of thyroid state on the enzymatic characteristic of cardiac myosin: a difference in behavior of rat and rabbit cardiac myosin. Circ. Res. 36:208-215.

5. Yazaki, Y., S. Ueda, R. Nagai, and K. Shimada. 1979. Cardiac atrial myosin adenosine triphosphatase of animals and humans: distinctive enzymatic properties compared with cardiac ventricular myosin. Circ. Res. 45:522-527.

6. Alpert, N. R., and L. A. Murieri. 1982. Increased myothermal economy of isometric force generation in compensated cardiac hypertrophy induced by pulmonary artery constriction in the rabbit. Circ. Res. 50:491-500.

7. Ebrecht, G., H. Rupp, and R. Jacob. 1982. Alteration of mechanical parameters in chemically skinned preparations of rat myocardium as a function of isoenzyme pattern of myosin. Basic Res. Cardiol. 77:220234.

8. Sartore, S., L. Gorza, S. Pierobon Bormioli, L. Dalla Libera, and S. Schaffino. 1981. Myosin types and fiber types in cardiac muscle. I. Ventricular myocardium. J. Cell Biol. 88:226-233.

9. Gorza, L., S. Sartore, and S. Schaffino. 1982. Myosin types and fiber types in cardiac muscle. II. Atrial myocardium. J. Cell Biol. 95:838-845.

10. Sartore, S., S. Pierobon-Bormioli, and S. Schaffino. 1978. Immunohistochemical evidence for myosin polymorphism in the chicken heart. Nature (Lond.). 274:82-83.

11. Lompre, A. M., J. J. Mercadier, C. Wisnewsky, P. Bouveret, C.
Pantaloni, A. d'Albis, and K. Schwartz. 1981. Species- and age-dependent changes in the relative amounts of cardiac myosin isoenzymes in mammals. Dev. Biol. 84:286-290.

12. Banerjee, S. K., and J. Wiener. 1983. Effects of aging on atrial and ventricular human myosin. Basic Res. Cardiol. 78:685-694.

13. Chizzonite, R. A., A. W. Everett, W. A. Clark, S. Jacovcic, M. Rabinowitz, and R. Zak. 1982. Isolation and characterization of two molecular variants of myosin heavy chain from rabbit ventricle: change in their content during normal growth and after treatment with thyroid hormone. J. Biol. Chem. 257:2056-2065.

14. Lompre, A. M., L. K. Schwarz, A. d'Albis, G. Lacmbe, N. V. Thiem, and B. Swynghedaw. 1979. Myosin isozyme redistribution in chronic heart overload. Nature (Lond.). 282:105-107.

15. Gorza, L., P. Pauletto, A. C. Pessina, S. Sartore, and S. Schaffino. 1981. Isomyosin distribution in normal and pressure-overloaded rat ventricular myocardium: an immunohistochemical study. Circ. Res. 49:1003-1009.

16. Yazaki, Y., H. Tsuchimochi, M. Kuro-o, M. Kurabayashi, M. Isobe, S. Ueda, R. Nagai, and F. Takaku. 1984. Distribution of myosin isozymes in human atrial and ventricular myocardium: comparison in normal and overloaded heart. Eur. Heart. J. 5(Suppl. F):103-110.

17. Tsuchimochi, H., M. Sugi, M. Kuro-o, S. Ueda, F. Takaku, S. Furuta, T. Shirai, and Y. Yazaki. 1984. Isozymic changes in myosin of human atrial myocardium induced by overload: immunohistochemical study using monoclonal antibodies. J. Clin. Invest. 74:662-665.

18. Gorza, L., J. J. Mercadier, K. Schwartz, L. E. Thornell, S. Sartore, and S. Schaffino. 1984. Myosin types in the human heart: an immunofluorescence study of normal and hypertrophied atrial and ventricular myocardium. Circ. Res. 54:694-702.

19. Yazaki, Y., S. Mochinaga, and M. S. Raben. 1973. Fraction of the light chains from rat and rabbit cardiac myosin. Biochem. Biophys. Acta. 328:464-469.

20. Köhler, G., and C. Milstein. 1973. Continuous cultures of fused cells secreting antibody of predefined specificity. Nature (Lond.). 256:495-497.

21. Guesdon, J., T. Ternynck, and S. Avrameas. 1979. The use of avidin-biotin interaction in immunoenzymatic techniques. J. Histochem. Cytochem. 27:1131-1139.

22. Davies, M. J., R. H. Anderson, and A. E. Becker. 1983. Methods of studying the conduction system. In The Conduction System of the Heart. M. J. Davies, R. H. Anderson, and A. E. Becker, editors. Butterworth \& Co. Ltd., London. 324-330.

23. Thornell, L. E., A. Eriksson, T. Stigbrand, and M. Sjostrom. 1978. Structural proteins in cow Purkinje and ordinary ventricular fibers: a marked difference. J. Mol. Cell. Cardiol. 10:605-616.

24. Tamura, Y., T. Nishimoto, S. Miyakami, H. Sako, K. Saito, and H. Mori. 1984. Characteristics of ATPase activity and the subunit composition of myosin in the conduction system of bovine heart. FEBS (Fed. Eur. Biochem. Soc.) Lett. 178:209-212.

25. Saito, K., Y. Tamura, M. Saito, K. Matsumura, T. Niki, and H. Mori. 1981. Comparison of the subunit compositions and ATPase activities of myosin in the myocardium and conduction system. J. Mol. Cell. Cardiol. 13:311-322.

26. Sako, H., Y. Tamura, S. Miyakami, K. Saito, T. Nishimoto, N. Nagase, S. Kobayashi, T. Niki, and H. Mori. 1984. Comparison of subunit compositions and isoenzymes of myosin in atrium, ventricle, and conduction system (abstr). Jpn. Circ. J. 48:925. (Abstr.)

27. Gonzalez-Sanchez, A., and D. Bader. 1985. Characterization of a myosin heavy chain in the conductive system of the adult and developing chicken heart. J. Cell Biol. 100:270-275. 